\title{
MATRIX VARIATE KUMMER-DIRICHLET DISTRIBUTIONS
}

\author{
ARJUN K. GUPTA, LILIAM CARDEÑO, AND DAYA K. NAGAR
}

Received 15 July 2000 and in revised form 8 June 2001

The multivariate Kummer-Beta and multivariate Kummer-Gamma families of distributions have been proposed and studied recently by $\mathrm{Ng}$ and Kotz. These distributions are extensions of Kummer-Beta and Kummer-Gamma distributions. In this article we propose and study matrix variate generalizations of multivariate Kummer-Beta and multivariate Kummer-Gamma families of distributions.

\section{Introduction}

The Kummer-Beta and Kummer-Gamma families of distributions are defined by the density functions

$$
\begin{aligned}
& \frac{\Gamma(\alpha+\beta)}{\Gamma(\alpha) \Gamma(\beta)}\left\{{ }_{1} \mathrm{~F}_{1}(\alpha ; \alpha+\beta ;-\lambda)\right\}^{-1} \exp (-\lambda u) u^{\alpha-1}(1-u)^{\beta-1}, \quad 0<u<1 \\
& \{\Gamma(\alpha) \Psi(\alpha, \alpha-\gamma+1 ; \xi)\}^{-1} \exp (-\xi \nu) v^{\alpha-1}(1+v)^{-\gamma}, \quad v>0,
\end{aligned}
$$

respectively, where $\alpha>0, \beta>0, \xi>0,-\infty<\gamma, \lambda<\infty,{ }_{1} F_{1}$, and $\Psi$ are confluent hypergeometric functions. These distributions are extensions of Gamma and Beta distributions, and for $\alpha<1$ (and certain values of $\lambda$ and $\gamma$ ) yield bimodal distributions on finite and infinite ranges, respectively. These distributions are used (i) in the Bayesian analysis of queueing system where posterior distribution of certain basic parameters in $M / M / \infty$ queueing system is Kummer-Gamma and (ii) in common value auctions where the posterior distribution of "value of a single good" is Kummer-Beta. For properties and applications of these distributions the reader is referred to $\mathrm{Ng}$ and Kotz [7], Armero and Bayarri [1], and Gordy [2]. 
As the corresponding multivariate generalization of these distributions, we have the following $n$-dimensional densities:

$$
\begin{gathered}
\frac{\Gamma\left(\sum_{i=1}^{n} \alpha_{i}+\beta\right)}{\prod_{i=1}^{n} \Gamma\left(\alpha_{i}\right) \Gamma(\beta)}\left\{{ }_{1} F_{1}\left(\sum_{i=1}^{n} \alpha_{i} ; \sum_{i=1}^{n} \alpha_{i}+\beta ;-\lambda\right)\right\}^{-1} \exp \left(-\lambda \sum_{i=1}^{n} u_{i}\right) \\
\times \prod_{i=1}^{n} u_{i}^{\alpha_{i}-1}\left(1-\sum_{i=1}^{n} u_{i}\right)^{\beta-1}, \quad 0<u_{i}<1, \sum_{i=1}^{n} u_{i}<1,
\end{gathered}
$$

where $\alpha_{i}>0, i=1, \ldots, n, \beta>0,-\infty<\lambda<\infty$, and

$$
\begin{gathered}
\left\{\Gamma\left(\sum_{i=1}^{n} \alpha_{i}\right) \Psi\left(\sum_{i=1}^{n} \alpha_{i}, \sum_{i=1}^{n} \alpha_{i}-\gamma+1 ; \xi\right)\right\}^{-1} \exp \left(-\xi \sum_{i=1}^{n} v_{i}\right) \\
\times \prod_{i=1}^{n} v_{i}^{\alpha_{i}-1}\left(1+\sum_{i=1}^{n} v_{i}\right)^{-\gamma}, \quad v_{i}>0,
\end{gathered}
$$

where $\alpha_{i}>0, i=1, \ldots, n, \xi>0,-\infty<\gamma<\infty$, respectively. These distributions have been considered by $\mathrm{Ng}$ and Kotz [7] who refer to (1.3) and (1.4) as multivariate Kummer-Beta and multivariate Kummer-Gamma distributions, respectively. For $\lambda=0,(1.1)$ and (1.3) reduce to Beta and Dirichlet distributions with probability density functions

$$
\begin{gathered}
\frac{\Gamma(\alpha+\beta)}{\Gamma(\alpha) \Gamma(\beta)} u^{\alpha-1}(1-u)^{\beta-1}, \quad 0<u<1, \\
\frac{\Gamma\left(\sum_{i=1}^{n} \alpha_{i}+\beta\right)}{\prod_{i=1}^{n} \Gamma\left(\alpha_{i}\right) \Gamma(\beta)} \prod_{i=1}^{n} u_{i}^{\alpha_{i}-1}\left(1-\sum_{i=1}^{n} u_{i}\right)^{\beta-1}, \quad 0<u_{i}<1, \sum_{i=1}^{n} u_{i}<1,
\end{gathered}
$$

respectively. Since (1.3) is an extension of Dirichlet distribution and a multivariate generalization of Kummer-Beta distribution, an appropriate nomenclature for this distribution would be Kummer-Dirichlet distribution. In the same vein, we may call (1.4) a Kummer-Dirichlet distribution. Further, in order to distinguish between these two distributions ((1.3) and (1.4)), we call them Kummer-Dirichlet type I and Kummer-Dirichlet type II distributions.

In this article we propose and study matrix variate generalizations of (1.3) and (1.4), respectively.

\section{Matrix variate Kummer-Dirichlet distributions}

We begin with a brief review of some definitions and notations. We adhere to standard notations (cf. Gupta and Nagar [3]). Let $A=\left(a_{i j}\right)$ be a $p \times p$ matrix. 
Then, $A^{\prime}$ denotes the transpose of $A ; \operatorname{tr}(A)=a_{11}+\cdots+a_{p p}$; etr $(A)=$ $\exp (\operatorname{tr}(A)) ; \operatorname{det}(A)=\operatorname{determinant}$ of $A ; A>0$ means that $A$ is symmetric positive definite and $A^{1 / 2}$ denotes the unique symmetric positive definite square root of $A>0$. The multivariate gamma function $\Gamma_{p}(m)$ is defined as

$$
\Gamma_{p}(m)=\pi^{p(p-1) / 4} \prod_{j=1}^{p} \Gamma\left(m-\frac{j-1}{2}\right), \quad \operatorname{Re}(m)>\frac{p-1}{2},
$$

where $\operatorname{Re}(\cdot)$ denotes the real part of $(\cdot)$. It is straightforward to show that

$$
\Gamma_{p}(m)=\int_{R>0} \operatorname{det}(R)^{m-(p+1) / 2} \operatorname{etr}(-R) d R, \quad \operatorname{Re}(m)>\frac{p-1}{2},
$$

where the integral has been evaluated over the space of the $p \times p$ symmetric positive definite matrices. The integral representation of the confluent hypergeometric function ${ }_{1} F_{1}$ is given by

$$
\begin{aligned}
{ }_{1} F_{1}(a ; b ; X)= & \frac{\Gamma_{p}(b)}{\Gamma_{p}(a) \Gamma_{p}(b-a)} \\
& \times \int_{0<R<I_{p}} \operatorname{det}(R)^{a-(p+1) / 2} \operatorname{det}\left(I_{p}-R\right)^{b-a-(p+1) / 2} \operatorname{etr}(X R) d R,
\end{aligned}
$$

where $\operatorname{Re}(a)>(p-1) / 2$ and $\operatorname{Re}(b-a)>(p-1) / 2$. The confluent hypergeometric function $\Psi$ of a $p \times p$ symmetric matrix $X$ is defined by

$$
\begin{aligned}
\Psi(a, c ; X)= & \frac{1}{\Gamma_{p}(a)} \\
& \times \int_{R>0} \operatorname{etr}(-X R) \operatorname{det}(R)^{a-(p+1) / 2} \operatorname{det}\left(I_{p}+R\right)^{c-a-(p+1) / 2} d R,
\end{aligned}
$$

where $\operatorname{Re}(X)>0$ and $\operatorname{Re}(a)>(p-1) / 2$.

Now we define the corresponding matrix variate generalizations of (1.3) and (1.4) as follows.

Definition 2.1. The $p \times p$ symmetric positive definite random matrices $\mathrm{U}_{1}, \ldots$, $\mathrm{U}_{\mathrm{n}}$ are said to have the matrix variate Kummer-Dirichlet type I distribution with parameters $\alpha_{1}, \ldots, \alpha_{n}, \beta$ and $\Lambda$, denoted by $\left(\mathrm{U}_{1}, \ldots, \mathrm{U}_{n}\right) \sim$ $\operatorname{KD}_{p}^{I}\left(\alpha_{1}, \ldots, \alpha_{n}, \beta, \Lambda\right)$, if their joint probability density function (pdf) is given by 


$$
\begin{gathered}
\mathrm{K}_{1}\left(\alpha_{1}, \ldots, \alpha_{n}, \beta, \Lambda\right) \operatorname{etr}\left(-\Lambda \sum_{i=1}^{n} \mathrm{u}_{i}\right) \\
\times \prod_{i=1}^{n} \operatorname{det}\left(\mathrm{U}_{i}\right)^{\alpha_{i}-(p+1) / 2} \operatorname{det}\left(\mathrm{I}_{p}-\sum_{i=1}^{n} \mathrm{U}_{i}\right)^{\beta-(p+1) / 2}, \\
0<\mathrm{U}_{i}<\mathrm{I}_{p}, 0<\sum_{i=1}^{n} \mathrm{u}_{i}<\mathrm{I}_{p},
\end{gathered}
$$

where $\alpha_{i}>(p-1) / 2, i=1, \ldots, n, \beta>(p-1) / 2, \Lambda(p \times p)$ is symmetric and $\mathrm{K}_{1}\left(\alpha_{1}, \ldots, \alpha_{n}, \beta, \Lambda\right)$ is the normalizing constant.

Definition 2.2. The $p \times p$ symmetric positive definite random matrices $V_{1}, \ldots$, $\mathrm{V}_{\mathrm{n}}$ are said to have the matrix variate Kummer-Dirichlet type II distribution with parameters $\alpha_{1}, \ldots, \alpha_{n}, \gamma$ and $\Xi$, denoted by $\left(V_{1}, \ldots, V_{n}\right) \sim \operatorname{KD}_{p}^{I I}\left(\alpha_{1}, \ldots\right.$, $\left.\alpha_{n}, \gamma, \Xi\right)$, if their joint pdf is given by

$$
\begin{aligned}
& \mathrm{K}_{2}\left(\alpha_{1}, \ldots, \alpha_{n}, \gamma, \Xi\right) \operatorname{etr}\left(-\Xi \sum_{i=1}^{n} \mathrm{~V}_{i}\right) \\
& \times \prod_{i=1}^{n} \operatorname{det}\left(\mathrm{V}_{i}\right)^{\alpha_{i}-(p+1) / 2} \operatorname{det}\left(\mathrm{I}_{p}+\sum_{i=1}^{n} \mathrm{~V}_{i}\right)^{-\gamma}, \quad \mathrm{V}_{i}>0,
\end{aligned}
$$

where $\alpha_{i}>(p-1) / 2, i=1, \ldots, n,-\infty<\gamma<\infty, \Xi(p \times p)>0$, and $\mathrm{K}_{2}\left(\alpha_{1}, \ldots, \alpha_{n}, \gamma, \Xi\right)$ is the normalizing constant.

The normalizing constants in (2.5) and (2.6) are given as

$$
\begin{aligned}
& \left\{K_{1}\left(\alpha_{1}, \ldots, \alpha_{n}, \beta, \Lambda\right)\right\}^{-1} \\
& =\int_{\substack{n \\
i=1 \\
\mathrm{U}_{i}>0}} \ldots \int_{\substack{\mathrm{U}_{i}<\mathrm{I}_{p} \\
\text { in }}} \operatorname{etr}\left(-\Lambda \sum_{i=1}^{n} \mathrm{u}_{i}\right) \\
& \times \prod_{i=1}^{n} \operatorname{det}\left(\mathrm{U}_{i}\right)^{\alpha_{i}-(p+1) / 2} \operatorname{det}\left(\mathrm{I}_{p}-\sum_{i=1}^{n} \mathrm{U}_{i}\right)^{\beta-(p+1) / 2} \prod_{i=1}^{n} d u_{i} \\
& =\frac{\prod_{i=1}^{n} \Gamma_{p}\left(\alpha_{i}\right)}{\Gamma_{p}\left(\sum_{i=1}^{n} \alpha_{i}\right)} \int_{0<U<I_{p}} \operatorname{etr}(-\Lambda U) \operatorname{det}(U)^{\sum_{i=1}^{n} \alpha_{i}-(p+1) / 2} \\
& \times \operatorname{det}\left(I_{p}-U\right)^{\beta-(p+1) / 2} d U
\end{aligned}
$$




$$
=\frac{\prod_{i=1}^{n} \Gamma_{p}\left(\alpha_{i}\right) \Gamma_{p}(\beta)}{\Gamma_{p}\left(\sum_{i=1}^{n} \alpha_{i}+\beta\right)}{ }_{1} F_{1}\left(\sum_{i=1}^{n} \alpha_{i} ; \sum_{i=1}^{n} \alpha_{i}+\beta ;-\Lambda\right),
$$

$$
\begin{aligned}
\left\{\mathrm{K}_{2}\left(\alpha_{1}, \ldots, \alpha_{n}, \gamma, \Xi\right)\right\}^{-1} & =\int_{V_{1}>0} \ldots \int_{V_{n}>0} \operatorname{etr}\left(-\Xi \sum_{i=1}^{n} V_{i}\right) \\
& \times \prod_{i=1}^{n} \operatorname{det}\left(V_{i}\right)^{\alpha_{i}-(p+1) / 2} \operatorname{det}\left(I_{p}+\sum_{i=1}^{n} V_{i}\right)^{-\gamma} \prod_{i=1}^{n} d V_{i} \\
= & \frac{\prod_{i=1}^{n} \Gamma_{p}\left(\alpha_{i}\right)}{\Gamma_{p}\left(\sum_{i=1}^{n} \alpha_{i}\right)} \int_{V>0} \operatorname{etr}(-\Xi V) \operatorname{det}(V)^{\sum_{i=1}^{n} \alpha_{i}-(p+1) / 2} \operatorname{det}\left(I_{p}+V\right)^{-\gamma} d V \\
= & \prod_{i=1}^{n} \Gamma_{p}\left(\alpha_{i}\right) \Psi\left(\sum_{i=1}^{n} \alpha_{i}, \sum_{i=1}^{n} \alpha_{i}-\gamma+\frac{p+1}{2} ; \Xi\right),
\end{aligned}
$$

respectively, where ${ }_{1} \mathrm{~F}_{1}$ and $\Psi$ are confluent hypergeometric functions of matrix argument.

For $\Lambda=0$, the matrix variate Kummer-Dirichlet type I distribution collapses to an ordinary matrix variate Dirichlet type I distribution with pdf

$$
\begin{gathered}
\frac{\Gamma_{p}\left(\sum_{i=1}^{n} \alpha_{i}+\beta\right)}{\prod_{i=1}^{n} \Gamma_{p}\left(\alpha_{i}\right) \Gamma_{p}(\beta)} \prod_{i=1}^{n} \operatorname{det}\left(\mathrm{U}_{i}\right)^{\alpha_{i}-(p+1) / 2} \operatorname{det}\left(\mathrm{I}_{p}-\sum_{i=1}^{n} \mathrm{u}_{i}\right)^{\beta-(p+1) / 2}, \\
0<\mathrm{U}_{i}<\mathrm{I}_{\mathfrak{p}}, 0<\sum_{i=1}^{n} \mathrm{U}_{i}<\mathrm{I}_{\mathrm{p}},
\end{gathered}
$$

where $\alpha_{i}>(p-1) / 2, i=1, \ldots, n$, and $\beta>(p-1) / 2$. A common notation to designate that $\left(\mathrm{U}_{1}, \ldots, \mathrm{U}_{\mathrm{n}}\right)$ has this density is $\left(\mathrm{U}_{1}, \ldots, \mathrm{U}_{\mathrm{n}}\right) \sim$ $\mathrm{D}_{\mathfrak{p}}^{\mathrm{I}}\left(\alpha_{1}, \ldots, \alpha_{\mathfrak{n}} ; \beta\right)$. For $\gamma=0$, the matrix variate Kummer-Dirichlet type II density simplifies to the product of $\mathrm{n}$ matrix variate Gamma densities.

For $p=1$, the densities in (2.5) and (2.6) simplify to Kummer-Dirichlet type I (multivariate Kummer-Beta) and Kummer-Dirichlet type II (multivariate Kummer-Gamma) densities, respectively. For $n=1$, the matrix variate Kummer-Dirichlet type I and matrix variate Kummer-Dirichlet type II distributions reduce to the matrix variate Kummer-Beta and matrix variate Kummer-Gamma distributions, respectively. These two distributions have been studied by Nagar and Gupta [6] and Nagar and Cardeño [5]. Substituting 
$n=1$ in (2.5) and (2.6), the matrix variate Kummer-Beta and matrix variate Kummer-Gamma densities are obtained as

$$
\begin{aligned}
& \mathrm{K}_{1}(\alpha, \beta, \Lambda) \operatorname{etr}(-\Lambda \mathrm{U}) \operatorname{det}(\mathrm{U})^{\alpha-(\mathrm{p}+1) / 2} \\
& \quad \times \operatorname{det}\left(\mathrm{I}_{\mathrm{p}}-\mathrm{U}\right)^{\beta-(\mathrm{p}+1) / 2}, \quad 0<\mathrm{U}<\mathrm{I}_{\mathrm{p}}, \\
& \mathrm{K}_{2}(\alpha, \gamma, \Xi) \operatorname{etr}(-\Xi \mathrm{V}) \operatorname{det}(\mathrm{V})^{\alpha-(\mathrm{p}+1) / 2} \operatorname{det}\left(\mathrm{I}_{\mathrm{p}}+\mathrm{V}\right)^{-\gamma}, \quad \mathrm{V}>0,
\end{aligned}
$$

respectively, where $\alpha>(p-1) / 2, \beta>(p-1) / 2,-\infty<\gamma<\infty, \Lambda=\Lambda^{\prime}$, and $\Xi(p \times p)>0$. These two distributions are designated by $\mathrm{U} \sim \mathrm{KB}_{\mathrm{p}}(\alpha, \beta, \Lambda)$ and $\mathrm{V} \sim \mathrm{KG}_{\mathrm{p}}(\alpha, \gamma, \Xi)$. It may be noted that the matrix variate Kummer-Dirichlet distributions are special cases of the matrix variate Liouville distribution.

Using certain transformations, generalized matrix variate Kummer-Dirichlet distributions are generated as given in the next two theorems.

Theorem 2.3. Let $\left(\mathrm{U}_{1}, \ldots, \mathrm{U}_{n}\right) \sim \mathrm{KD}_{\mathrm{p}}^{\mathrm{I}}\left(\alpha_{1}, \ldots, \alpha_{n}, \beta, \Lambda\right)$ and $\Psi_{1}, \ldots, \Psi_{n}, \Omega$ be symmetric matrices such that $\Omega>0$ and $\Omega-\sum_{i=1}^{n} \Psi_{i}>0$. Define

$$
Z_{i}=\left(\Omega-\sum_{i=1}^{n} \Psi_{i}\right)^{1 / 2} U_{i}\left(\Omega-\sum_{i=1}^{n} \Psi_{i}\right)^{1 / 2}+\Psi_{i}, \quad i=1, \ldots, n .
$$

Then $\left(Z_{1}, \ldots, Z_{n}\right)$ have the generalized matrix variate Kummer-Dirichlet type I distribution with $p d f$

$$
\begin{aligned}
& \frac{K_{1}\left(\alpha_{1}, \ldots, \alpha_{n}, \beta, \Lambda\right)}{\operatorname{det}\left(\Omega-\sum_{i=1}^{n} \Psi_{i}\right)^{\sum_{i=1}^{r} \alpha_{i}+\beta-(p+1) / 2}} \\
& \times \frac{\prod_{i=1}^{n} \operatorname{det}\left(Z_{i}-\Psi_{i}\right)^{\alpha_{i}-(p+1) / 2} \operatorname{det}\left(\Omega-\sum_{i=1}^{n} Z_{i}\right)^{\beta-(p+1) / 2}}{\operatorname{etr}\left\{\left(\Omega-\sum_{i=1}^{n} \Psi_{i}\right)^{-1 / 2} \Lambda\left(\Omega-\sum_{i=1}^{n} \Psi_{i}\right)^{-1 / 2} \sum_{i=1}^{n}\left(Z_{i}-\Psi_{i}\right)\right\}}, \\
& \Psi_{i}<Z_{i}<\Omega, i=1, \ldots, n, \sum_{i=1}^{n} Z_{i}<\Omega .
\end{aligned}
$$

Proof. Making the transformation $\mathrm{U}_{i}=\left(\Omega-\sum_{i=1}^{n} \Psi_{i}\right)^{-1 / 2}\left(Z_{i}-\Psi_{i}\right)(\Omega-$ $\left.\sum_{i=1}^{r} \Psi_{i}\right)^{-1 / 2}, i=1, \ldots, n$, with Jacobian $\mathrm{J}\left(\mathrm{U}_{1}, \ldots, \mathrm{U}_{\mathrm{n}} \rightarrow \mathrm{Z}_{1}, \ldots, \mathrm{Z}_{\mathrm{n}}\right)=$ $\operatorname{det}\left(\Omega-\sum_{i=1}^{n} \Psi_{i}\right)^{-n(p+1) / 2}$ in (2.5), we get (2.12).

If $\left(Z_{1}, \ldots, Z_{n}\right)$ has the pdf (2.12), then we write $\left(Z_{1}, \ldots, Z_{n}\right) \sim G D_{p}^{I}\left(\alpha_{1}\right.$, $\left.\ldots, \alpha_{n}, \beta, \Lambda ; \Omega ; \Psi_{1}, \ldots, \Psi_{n}\right)$. Note that $\operatorname{GKD}_{p}^{I}\left(\alpha_{1}, \ldots, \alpha_{n}, \beta, \wedge ; I_{p} ; 0, \ldots, 0\right) \equiv$ $\operatorname{KD}_{p}^{\mathrm{I}}\left(\alpha_{1}, \ldots, \alpha_{\mathrm{n}}, \beta, \Lambda\right)$. 
Theorem 2.4. Let $\left(\mathrm{V}_{1}, \ldots, \mathrm{V}_{\mathrm{n}}\right) \sim \mathrm{KD}_{\mathrm{p}}^{\mathrm{II}}\left(\alpha_{1}, \ldots, \alpha_{n}, \gamma, \Xi\right)$ and $\Psi_{1}, \ldots, \Psi_{n}, \Omega$ be symmetric matrices such that $\Omega>0$ and $\Omega+\sum_{i=1}^{n} \Psi_{i}>0$. Define

$$
Y_{i}=\left(\Omega+\sum_{i=1}^{n} \Psi_{i}\right)^{1 / 2} V_{i}\left(\Omega+\sum_{i=1}^{n} \Psi_{i}\right)^{1 / 2}+\Psi_{i}, \quad i=1, \ldots, n
$$

Then, $\left(\mathrm{Y}_{1}, \ldots, \mathrm{Y}_{\mathrm{n}}\right)$ have the generalized matrix variate Kummer-Dirichlet type II distribution with $p d f$

$$
\begin{aligned}
& \frac{K_{2}\left(\alpha_{1}, \ldots, \alpha_{n}, \gamma, \Xi\right)}{\operatorname{det}\left(\Omega+\sum_{i=1}^{n} \Psi_{i}\right)^{\sum_{i=1}^{n} \alpha_{i}-\gamma}} \\
& \quad \times \frac{\prod_{i=1}^{n} \operatorname{det}\left(Y_{i}-\Psi_{i}\right)^{\alpha_{i}-(p+1) / 2} \operatorname{det}\left(\Omega+\sum_{i=1}^{n} Y_{i}\right)^{-\gamma}}{\operatorname{etr}\left\{\left(\Omega+\sum_{i=1}^{n} \Psi_{i}\right)^{-1 / 2} \Xi\left(\Omega+\sum_{i=1}^{n} \Psi_{i}\right)^{-1 / 2} \sum_{i=1}^{n}\left(Y_{i}-\Psi_{i}\right)\right\}}, \\
& Y_{i}>\Psi_{i}, i=1, \ldots, n .
\end{aligned}
$$

Proof. Making the transformation $V_{i}=\left(\Omega+\sum_{i=1}^{n} \Psi_{i}\right)^{-1 / 2}\left(Y_{i}-\Psi_{i}\right)(\Omega+$ $\left.\sum_{i=1}^{n} \Psi_{i}\right)^{-1 / 2}, i=1, \ldots, n$, with the Jacobian $J\left(V_{1}, \ldots, V_{n} \rightarrow Y_{1}, \ldots, Y_{n}\right)=$ $\operatorname{det}\left(\Omega+\sum_{i=1}^{n} \Psi_{i}\right)^{-n(p+1) / 2}$ in (2.6), we get (2.14).

If $\left(Y_{1}, \ldots, Y_{n}\right)$ has pdf (2.14), then we write $\left(Y_{1}, \ldots, Y_{n}\right) \sim \operatorname{GKD}_{p}^{I I}\left(\alpha_{1}\right.$, $\left.\ldots, \alpha_{n}, \gamma, \Xi ; \Omega ; \Psi_{1}, \ldots, \Psi_{n}\right)$. In this case $\operatorname{GKD}_{p}^{I I}\left(\alpha_{1}, \ldots, \alpha_{n}, \gamma ; I_{p} ; 0, \ldots, 0\right) \equiv$ $\operatorname{KD}_{\mathrm{p}}^{\mathrm{II}}\left(\alpha_{1}, \ldots, \alpha_{n} ; \gamma, \Xi\right)$.

\section{Properties}

In this section, we study certain properties of matrix variate KummerDirichlet type I and II distributions. It may be noted that for $\Lambda=\lambda \mathrm{I}_{p}$, $\Xi=\xi I_{p}$ densities (2.5) and (2.6) are orthogonally invariant. That is, for any fixed orthogonal matrix $\Gamma(p \times p)$, the distribution of $\left(\Gamma U_{1} \Gamma^{\prime}, \ldots, \Gamma U_{n} \Gamma^{\prime}\right)$ is the same as the distribution of $\left(\mathrm{U}_{1}, \ldots, \mathrm{U}_{n}\right)$, and similarly the distribution of $\left(\Gamma \mathrm{V}_{1} \Gamma^{\prime}, \ldots, \Gamma \mathrm{V}_{n} \Gamma^{\prime}\right)$ is the same as that of $\left(\mathrm{V}_{1}, \ldots, \mathrm{V}_{\mathrm{n}}\right)$. Our next two results give marginal and conditional distributions.

Theorem 3.1. If $\left(\mathrm{U}_{1}, \ldots, \mathrm{U}_{\mathrm{n}}\right) \sim \mathrm{KD}_{\mathrm{p}}^{\mathrm{I}}\left(\alpha_{1}, \ldots, \alpha_{\mathrm{n}}, \beta, \Lambda\right)$, then the joint marginal pdf of $\mathrm{U}_{1}, \ldots, \mathrm{U}_{\mathrm{m}}, \mathrm{m} \leq \mathrm{n}$, is given by 


$$
\begin{gathered}
\mathrm{K}_{1}\left(\alpha_{1}, \ldots, \alpha_{\mathrm{m}}, \sum_{i=\mathrm{m}+1}^{n} \alpha_{i}+\beta, \Lambda\right) \operatorname{etr}\left(-\Lambda \sum_{i=1}^{m} \mathrm{u}_{i}\right) \\
\times \prod_{i=1}^{\mathrm{m}} \operatorname{det}\left(\mathrm{u}_{i}\right)^{\alpha_{i}-(p+1) / 2} \operatorname{det}\left(\mathrm{I}_{p}-\sum_{i=1}^{m} \mathrm{u}_{i}\right)^{\sum_{i=m+1}^{n} \alpha_{i}+\beta-(p+1) / 2} \\
\times{ }_{1} \mathrm{~F}_{1}\left(\sum_{i=m+1}^{n} \alpha_{i} ; \sum_{i=m+1}^{n} \alpha_{i}+\beta ;-\Lambda\left(\mathrm{I}_{p}-\sum_{i=1}^{m} \mathrm{u}_{i}\right)\right) \\
0<\mathrm{U}_{i}<\mathrm{I}_{p}, 0<\sum_{i=1}^{m} \mathrm{u}_{i}<\mathrm{I}_{p}
\end{gathered}
$$

and the conditional density of $\left(\mathrm{U}_{\mathrm{m}+1}, \ldots, \mathrm{U}_{\mathrm{n}}\right) \mid\left(\mathrm{U}_{1}, \ldots, \mathrm{U}_{\mathrm{m}}\right)$ is given by

$$
\begin{aligned}
& \frac{\mathrm{K}_{1}\left(\alpha_{1}, \ldots, \alpha_{n}, \beta, \Lambda\right)}{\mathrm{K}_{1}\left(\alpha_{1}, \ldots, \alpha_{m}, \sum_{i=m+1}^{n} \alpha_{i}+\beta, \Lambda\right)} \\
& \times \frac{\operatorname{etr}\left(-\Lambda \sum_{i=m+1}^{n} \mathrm{U}_{i}\right)}{\operatorname{det}\left(\mathrm{I}_{p}-\sum_{i=1}^{m} \mathrm{U}_{i}\right)^{\sum_{i=m+1}^{n} \alpha_{i}+\beta-(p+1) / 2}} \\
& \times \frac{\prod_{i=m+1}^{n} \operatorname{det}\left(\mathrm{U}_{i}\right)^{\alpha_{i}-(p+1) / 2} \operatorname{det}\left(\mathrm{I}_{p}-\sum_{i=1}^{m} \mathrm{U}_{i}-\sum_{i=m+1}^{n} \mathrm{U}_{i}\right)^{\beta-(p+1) / 2}}{{ }_{1} \mathrm{~F}_{1}\left(\sum_{i=m+1}^{n} \alpha_{i} ; \sum_{i=m+1}^{n} \alpha_{i}+\beta ;-\Lambda\left(\mathrm{I}_{p}-\sum_{i=1}^{m} \mathrm{U}_{i}\right)\right)}, \\
& \quad 0<\mathrm{U}_{i}<\mathrm{I}_{p}-\sum_{i=1}^{m} \mathrm{U}_{i}, i=m+1, \ldots, n, \sum_{i=m+1}^{n} \mathrm{u}_{i}<\mathrm{I}_{p}-\sum_{i=1}^{m} \mathrm{U}_{i} .
\end{aligned}
$$

Proof. First we find the marginal density of $\mathrm{u}_{1}, \ldots, \mathrm{u}_{\mathrm{n}-1}$ by integrating out $\mathrm{u}_{\mathrm{n}}$ from the joint density of $\mathrm{U}_{1}, \ldots, \mathrm{u}_{\mathrm{n}}$ as

$$
\begin{aligned}
& \mathrm{K}_{1}\left(\alpha_{1}, \ldots, \alpha_{n}, \beta, \Lambda\right) \int_{0<\mathrm{U}_{n}<\mathrm{I}_{p}-\sum_{i=1}^{n-1} \mathrm{u}_{i}} \operatorname{etr}\left(-\Lambda \sum_{i=1}^{n} \mathrm{U}_{i}\right) \\
& \times \prod_{i=1}^{n} \operatorname{det}\left(\mathrm{U}_{i}\right)^{\alpha_{i}-(p+1) / 2} \operatorname{det}\left(\mathrm{I}_{p}-\sum_{i=1}^{n} \mathrm{U}_{i}\right)^{\beta-(p+1) / 2} \mathrm{du}_{n}
\end{aligned}
$$


Arjun K. Gupta et al. 125

Now, substituting $Z_{n}=\left(I_{p}-\sum_{i=1}^{n-1} U_{i}\right)^{-1 / 2} U_{n}\left(I_{p}-\sum_{i=1}^{n-1} U_{i}\right)^{-1 / 2}$ with Jacobian $\mathrm{J}\left(\mathrm{U}_{\mathrm{n}} \rightarrow \mathrm{Z}_{\mathrm{n}}\right)=\operatorname{det}\left(\mathrm{I}_{\mathrm{p}}-\sum_{i=1}^{n-1} \mathrm{U}_{i}\right)^{(p+1) / 2}$ in (3.2), we get

$$
\begin{aligned}
& \mathrm{K}_{1}\left(\alpha_{1}, \ldots, \alpha_{n}, \beta, \Lambda\right) \operatorname{etr}\left(-\Lambda \sum_{i=1}^{n-1} \mathrm{U}_{i}\right) \\
& \times \prod_{i=1}^{n-1} \operatorname{det}\left(\mathrm{U}_{i}\right)^{\alpha_{i}-(\mathrm{p}+1) / 2} \operatorname{det}\left(\mathrm{I}_{p}-\sum_{i=1}^{n-1} \mathrm{U}_{i}\right)^{\alpha_{n}+\beta-(p+1) / 2} \\
& \times \int_{0<Z_{n}<\mathrm{I}_{p}} \operatorname{etr}\left[-\left(\mathrm{I}_{p}-\sum_{i=1}^{n-1} \mathrm{U}_{i}\right)^{1 / 2} \wedge\left(\mathrm{I}_{p}-\sum_{i=1}^{n-1} \mathrm{U}_{i}\right)^{1 / 2} \mathrm{Z}_{\mathrm{n}}\right] \\
& \times \operatorname{det}\left(\mathrm{Z}_{n}\right)^{\alpha_{n}-(p+1) / 2} \operatorname{det}\left(\mathrm{I}_{p}-\mathrm{Z}_{n}\right)^{\beta-(p+1) / 2} \mathrm{~d} Z_{n} .
\end{aligned}
$$

But

$$
\begin{aligned}
& \mathrm{K}_{1}\left(\alpha_{1}, \ldots, \alpha_{n}, \beta, \Lambda\right) \\
& \times \int_{0<Z_{r}<I_{p}} \operatorname{etr}\left[-\left(I_{p}-\sum_{i=1}^{n-1} U_{i}\right)^{1 / 2} \wedge\left(I_{p}-\sum_{i=1}^{n-1} U_{i}\right)^{1 / 2} Z_{n}\right] \\
& \quad \times \operatorname{det}\left(Z_{n}\right)^{\alpha_{n}-(p+1) / 2} \operatorname{det}\left(I_{p}-Z_{n}\right)^{\beta-(p+1) / 2} d Z_{n} \\
& =K_{1}\left(\alpha_{1}, \ldots, \alpha_{n}, \beta, \Lambda\right) \frac{\Gamma_{p}\left(\alpha_{n}\right) \Gamma_{p}(\beta)}{\Gamma_{p}\left(\alpha_{n}+\beta\right)}{ }_{1} F_{1}\left(\alpha_{n} ; \alpha_{n}+\beta ;-\Lambda\left(I_{p}-\sum_{i=1}^{n-1} U_{i}\right)\right) \\
& =K_{1}\left(\alpha_{1}, \ldots, \alpha_{n-1}, \alpha_{n}+\beta, \Lambda\right)_{1} F_{1}\left(\alpha_{n} ; \alpha_{n}+\beta ;-\Lambda\left(I_{p}-\sum_{i=1}^{n-1} U_{i}\right)\right) .
\end{aligned}
$$

Hence, we get the joint density of $\left(\mathrm{U}_{1}, \ldots, \mathrm{U}_{\mathrm{n}-1}\right)$ as

$$
\begin{aligned}
& \mathrm{K}_{1}\left(\alpha_{1}, \ldots, \alpha_{n-1}, \alpha_{n}+\beta, \Lambda\right) \operatorname{etr}\left(-\Lambda \sum_{i=1}^{n-1} \mathrm{U}_{i}\right) \prod_{i=1}^{n-1} \operatorname{det}\left(\mathrm{U}_{i}\right)^{\alpha_{i}-(p+1) / 2} \\
& \times \operatorname{det}\left(\mathrm{I}_{p}-\sum_{i=1}^{n-1} \mathrm{U}_{i}\right)^{\alpha_{n}+\beta-(p+1) / 2}{ }_{1} \mathrm{~F}_{1}\left(\alpha_{n} ; \alpha_{n}+\beta ;-\Lambda\left(\mathrm{I}_{p}-\sum_{i=1}^{n-1} \mathrm{U}_{i}\right)\right) .
\end{aligned}
$$


Repeating this procedure $n-m$ times gives the marginal density of $\left(U_{1}, \ldots\right.$, $\left.\mathrm{u}_{\mathrm{m}}\right)$ as

$$
\begin{aligned}
& \mathrm{K}_{1}\left(\alpha_{1}, \ldots, \alpha_{\mathrm{m}}, \sum_{i=\mathrm{m}+1}^{n} \alpha_{i}+\beta, \Lambda\right) \operatorname{etr}\left(-\Lambda \sum_{i=1}^{m} \mathrm{u}_{i}\right) \\
& \quad \times \prod_{i=1}^{\mathrm{m}} \operatorname{det}\left(\mathrm{u}_{i}\right)^{\alpha_{i}-(p+1) / 2} \operatorname{det}\left(\mathrm{I}_{p}-\sum_{i=1}^{\mathrm{m}} \mathrm{u}_{i}\right)^{\sum_{i=m+1}^{n} \alpha_{i}+\beta-(p+1) / 2} \\
& \quad \times{ }_{1} \mathrm{~F}_{1}\left(\sum_{i=m+1}^{n} \alpha_{i} ; \sum_{i=m+1}^{n} \alpha_{i}+\beta ;-\Lambda\left(\mathrm{I}_{p}-\sum_{i=1}^{m} \mathrm{u}_{i}\right)\right)
\end{aligned}
$$

Now, the second part of the theorem follows immediately.

Corollary 3.2. If $\left(\mathrm{U}_{1}, \ldots, \mathrm{U}_{\mathrm{n}}\right) \sim \mathrm{KD}_{\mathrm{p}}^{\mathrm{I}}\left(\alpha_{1}, \ldots, \alpha_{\mathrm{n}}, \beta, \Lambda\right)$, then the marginal pdf of $\mathrm{U}_{i}, i=1, \ldots, n$ is given by

$$
\begin{aligned}
\mathrm{K}_{1}\left(\alpha_{i}, \sum_{j=1(\neq i)}^{n} \alpha_{j}+\beta, \Lambda\right) \operatorname{etr}\left(-\Lambda \mathrm{U}_{i}\right) \operatorname{det}\left(\mathrm{U}_{i}\right)^{\alpha_{i}-(p+1) / 2} \\
\quad \times \operatorname{det}\left(\mathrm{I}_{p}-\mathrm{U}_{i}\right)^{\sum_{j=1(\neq i)}^{n} \alpha_{j}+\beta-(p+1) / 2} \\
\quad \times{ }_{1} \mathrm{~F}_{1}\left(\sum_{j=1(\neq i)}^{n} \alpha_{j} ; \sum_{j=1(\neq i)}^{n} \alpha_{j}+\beta ;-\Lambda\left(\mathrm{I}_{p}-\mathrm{U}_{i}\right)\right), \quad 0<\mathrm{U}_{i}<\mathrm{I}_{p} .
\end{aligned}
$$

It is interesting to note that the marginal density of $\mathrm{u}_{i}$ does not belong to the Kummer-Beta family and differs by an additional factor containing confluent hypergeometric function ${ }_{1} \mathrm{~F}_{1}$.

In Theorem 3.3 we give results on marginal and conditional distributions for Kummer-Dirichlet type II distribution. Before doing so, we need to give an integral that will be used in the derivation of marginal distribution. From (2.6) and (2.8), we have

$$
\begin{aligned}
\int_{X>0} \int_{Y>0} & \operatorname{etr}[-\Xi(X+Y)] \operatorname{det}(Y)^{a_{1}-(p+1) / 2} \\
& \times \operatorname{det}(X)^{a_{2}-(p+1) / 2} \operatorname{det}\left(I_{p}+X+Y\right)^{-b} d X d Y \\
= & \Gamma_{p}\left(a_{1}\right) \Gamma_{p}\left(a_{2}\right) \Psi\left(a_{1}+a_{2}, a_{1}+a_{2}-b+\frac{p+1}{2} ; \Xi\right)
\end{aligned}
$$

where $\operatorname{Re}\left(a_{1}\right)>(p-1) / 2, \operatorname{Re}\left(a_{2}\right)>(p-1) / 2$ and $\operatorname{Re}(\Xi)>0$. Substituting 
$\mathrm{W}=\left(\mathrm{I}_{\mathrm{p}}+\mathrm{X}\right)^{-1 / 2} \mathrm{Y}\left(\mathrm{I}_{\mathrm{p}}+\mathrm{X}\right)^{-1 / 2}$ with the Jacobian $\mathrm{J}(\mathrm{Y} \rightarrow \mathrm{W})=\operatorname{det}\left(\mathrm{I}_{\mathrm{p}}+\right.$ $X)^{(p+1) / 2}$ in (3.9) and integrating $W$, we obtain

$$
\begin{aligned}
\int_{X>0} \operatorname{etr}(-\Xi X) \operatorname{det}(X)^{a_{2}-(p+1) / 2} \operatorname{det}\left(I_{p}+X\right)^{a_{1}-b} \\
\quad \times \Psi\left(a_{1}, a_{1}-b+\frac{p+1}{2} ; \Xi\left(I_{p}+X\right)\right) d X \\
=\Gamma_{p}\left(a_{2}\right) \Psi\left(a_{1}+a_{2}, a_{1}+a_{2}-b+\frac{p+1}{2} ; \Xi\right) .
\end{aligned}
$$

Now we turn to our problem of finding the marginal and conditional distributions.

Theorem 3.3. If $\left(\mathrm{V}_{1}, \ldots, \mathrm{V}_{\mathrm{n}}\right) \sim \mathrm{KD}_{\mathrm{p}}^{\mathrm{II}}\left(\alpha_{1}, \ldots, \alpha_{\mathrm{n}}, \gamma, \Xi\right)$, then the joint marginal pdf of $\mathrm{V}_{1}, \ldots, \mathrm{V}_{\mathrm{m}}, \mathrm{m} \leq \mathrm{n}$, is given by

$$
\begin{gathered}
\Gamma_{p}\left(\sum_{i=m+1}^{n} \alpha_{i}\right) K_{2}\left(\alpha_{1}, \ldots, \alpha_{m}, \sum_{i=m+1}^{n} \alpha_{i}, \gamma, \Xi\right) \operatorname{etr}\left(-\Xi \sum_{i=1}^{m} V_{i}\right) \\
\times \prod_{i=1}^{m} \operatorname{det}\left(V_{i}\right)^{\alpha_{i}-(p+1) / 2} \operatorname{det}\left(I_{p}+\sum_{i=1}^{m} V_{i}\right)^{-\gamma+\sum_{i=m+1}^{n} \alpha_{i}} \\
\times \Psi\left(\sum_{i=m+1}^{n} \alpha_{i}, \sum_{i=m+1}^{n} \alpha_{i}-\gamma+\frac{p+1}{2} ; \Xi\left(I_{p}+\sum_{j=1}^{m} V_{j}\right)\right), \\
V_{j}>0, j=1, \ldots, m,
\end{gathered}
$$

and the conditional density of $\left(\mathrm{V}_{\mathrm{m}+1}, \ldots, \mathrm{V}_{\mathrm{n}}\right) \mid\left(\mathrm{V}_{1}, \ldots, \mathrm{V}_{\mathrm{m}}\right)$ is given by

$$
\begin{aligned}
& \frac{\mathrm{K}_{2}\left(\alpha_{1}, \ldots, \alpha_{n}, \gamma, \Xi\right)}{\Gamma_{p}\left(\sum_{i=m+1}^{n} \alpha_{i}\right) K_{2}\left(\alpha_{1}, \ldots, \alpha_{m}, \sum_{i=m+1}^{n} \alpha_{i}, \gamma, \Xi\right)} \\
& \times \frac{\operatorname{etr}\left(-\Xi \sum_{i=m+1}^{n} V_{i}\right)}{\operatorname{det}\left(I_{p}+\sum_{i=1}^{m} V_{i}\right)^{-\gamma+\sum_{i=m+1}^{n} \alpha_{i}}} \\
& \times \frac{\prod_{i=m+1}^{n} \operatorname{det}\left(V_{i}\right)^{\alpha_{i}-(p+1) / 2} \operatorname{det}\left(I_{p}+\sum_{i=1}^{m} V_{i}+\sum_{i=m+1}^{n} V_{i}\right)^{-\gamma}}{\Psi\left(\sum_{i=m+1}^{n} \alpha_{i}, \sum_{i=m+1}^{n} \alpha_{i}-\gamma+(p+1) / 2 ; \Xi\left(I_{p}+\sum_{j=1}^{m} V_{j}\right)\right)}, \\
& \quad v_{i}>0, i=m+1, \ldots, n .
\end{aligned}
$$

Proof. In this case, to obtain the marginal density of $\mathrm{V}_{1}, \ldots, \mathrm{V}_{\mathrm{n}-1}$, we substitute $W_{n}=\left(I_{p}+\sum_{i=1}^{n-1} V_{i}\right)^{-1 / 2} V_{n}\left(I_{p}+\sum_{i=1}^{n-1} V_{i}\right)^{-1 / 2}$ with the 
Jacobian $J\left(V_{n} \rightarrow W_{n}\right)=\operatorname{det}\left(I_{p}+\sum_{i=1}^{n-1} V_{i}\right)^{(p+1) / 2}$. Thus, the joint density of $V_{1}, \ldots, V_{n-1}$ is obtained as

$$
\begin{aligned}
K_{2}\left(\alpha_{1}, \ldots, \alpha_{n}, \gamma, \Xi\right) \operatorname{etr}\left(-\Xi \sum_{i=1}^{n-1} V_{i}\right) \\
\quad \times \prod_{i=1}^{n-1} \operatorname{det}\left(V_{i}\right)^{\alpha_{i}-(p+1) / 2} \operatorname{det}\left(I_{p}+\sum_{i=1}^{n-1} V_{i}\right)^{-\gamma+\alpha_{n}} \\
\times \int_{W_{n}>0} \operatorname{etr}\left[-\left(I_{p}+\sum_{i=1}^{n-1} V_{i}\right)^{1 / 2} \Xi\left(I_{p}+\sum_{i=1}^{n-1} V_{i}\right)^{1 / 2} W_{n}\right] \\
=\Gamma_{p}\left(\alpha_{n}\right) K_{2}\left(\alpha_{1}, \ldots, \alpha_{n}, \gamma, \Xi\right) \operatorname{detr}\left(-\Xi \sum_{i=1}^{n-1} V_{i}\right)^{\alpha_{n}-(p+1) / 2} \operatorname{det}\left(I_{p}+W_{n}\right)^{-\gamma} d W_{n} \\
\times \prod_{i=1}^{n-1} \operatorname{det}\left(V_{i}\right)^{\alpha_{i}-(p+1) / 2} \operatorname{det}\left(I_{p}+\sum_{i=1}^{n-1} V_{i}\right)^{-\gamma+\alpha_{n}} \\
\times \Psi\left(\alpha_{n}, \alpha_{n}-\gamma+\frac{p+1}{2} ; \Xi\left(I_{p}+\sum_{i=1}^{n-1} V_{i}\right)\right)
\end{aligned}
$$

Further, substituting $W_{n-1}=\left(I_{p}+\sum_{i=1}^{n-2} V_{i}\right)^{-1 / 2} V_{n-1}\left(I_{p}+\sum_{i=1}^{n-2} V_{i}\right)^{-1 / 2}$ with the Jacobian $J\left(V_{n-1} \rightarrow W_{n-1}\right)=\operatorname{det}\left(I_{p}+\sum_{i=1}^{n-2} V_{i}\right)^{(p+1) / 2}$ in (3.13) and integrating $W_{n-1}$ using (3.10), we get the joint marginal density of $\mathrm{V}_{1}, \ldots, \mathrm{V}_{\mathrm{n}-2}$ as

$$
\begin{aligned}
& \Gamma_{p}\left(\alpha_{n}\right) K_{2}\left(\alpha_{1}, \ldots, \alpha_{n}, \gamma, \Xi\right) \operatorname{etr}\left(-\Xi \sum_{i=1}^{n-2} V_{i}\right) \\
& \times \prod_{i=1}^{n-2} \operatorname{det}\left(V_{i}\right)^{\alpha_{i}-(p+1) / 2} \operatorname{det}\left(I_{p}+\sum_{i=1}^{n-2} V_{i}\right)^{-\gamma+\alpha_{n}+\alpha_{n-1}} \\
& \times \int_{W_{n-1}>0} \operatorname{etr}\left[-\left(I_{p}+\sum_{i=1}^{n-2} V_{i}\right)^{1 / 2} \Xi\left(I_{p}+\sum_{i=1}^{n-2} V_{i}\right)^{1 / 2} W_{n-1}\right] \\
& \times \operatorname{det}\left(W_{n-1}\right)^{\alpha_{n-1}-(p+1) / 2} \operatorname{det}\left(I_{p}+W_{n-1}\right)^{-\gamma+\alpha_{n}}
\end{aligned}
$$




$$
\begin{gathered}
\times \Psi\left(\alpha_{n}, \alpha_{n}-\gamma+\frac{p+1}{2} ;\left(I_{p}+\sum_{i=1}^{n-2} V_{i}\right)^{1 / 2}\right. \\
\left.\times \Xi\left(I_{p}+\sum_{i=1}^{n-2} V_{i}\right)^{1 / 2} W_{n-1}\right) d W_{n-1} \\
=\Gamma_{p}\left(\alpha_{n}\right) \Gamma_{p}\left(\alpha_{n-1}\right) K_{2}\left(\alpha_{1}, \ldots, \alpha_{n}, \gamma, \Xi\right) \operatorname{etr}\left(-\Xi \sum_{i=1}^{n-2} V_{i}\right) \\
\times \prod_{i=1}^{n-2} \operatorname{det}\left(V_{i}\right)^{\alpha_{i}-(p+1) / 2} \operatorname{det}\left(I_{p}+\sum_{i=1}^{n-2} V_{i}\right)^{-\gamma+\alpha_{n}+\alpha_{n-1}} \\
\times \Psi\left(\alpha_{n}+\alpha_{n-1}, \alpha_{n}+\alpha_{n-1}-\gamma+\frac{p+1}{2} ; \Xi\left(I_{p}+\sum_{i=1}^{n-2} V_{i}\right)\right) .
\end{gathered}
$$

Integrating out $V_{n-2}, \ldots, V_{m+1}$ similarly, we get the marginal density of $\mathrm{V}_{1}, \ldots, \mathrm{V}_{\mathrm{m}}$ as

$$
\begin{aligned}
& \prod_{i=m+1}^{n} \Gamma_{p}\left(\alpha_{i}\right) K_{2}\left(\alpha_{1}, \ldots, \alpha_{n}, \gamma, \Xi\right) \operatorname{etr}\left(-\Xi \sum_{i=1}^{m} V_{i}\right) \\
& \quad \times \prod_{i=1}^{m} \operatorname{det}\left(V_{i}\right)^{\alpha_{i}-(p+1) / 2} \operatorname{det}\left(I_{p}+\sum_{i=1}^{m} V_{i}\right)^{-\gamma+\sum_{i=m+1}^{n} \alpha_{i}} \\
& \quad \times \Psi\left(\sum_{i=m+1}^{n} \alpha_{i}, \sum_{i=m+1}^{n} \alpha_{i}-\gamma+\frac{p+1}{2} ; \Xi\left(I_{p}+\sum_{i=1}^{m} V_{i}\right)\right)
\end{aligned}
$$

The final expression of the marginal density of $V_{1}, \ldots, V_{m}$ is obtained by noting that

$$
\begin{aligned}
& \prod_{i=m+1}^{n} \Gamma_{p}\left(\alpha_{i}\right) K_{2}\left(\alpha_{1}, \ldots, \alpha_{n}, \gamma, \Xi\right) \\
& \quad=\Gamma_{p}\left(\sum_{i=m+1}^{n} \alpha_{i}\right) K_{2}\left(\alpha_{1}, \ldots, \alpha_{m}, \sum_{i=m+1}^{n} \alpha_{i}, \gamma, \Xi\right) .
\end{aligned}
$$

The derivation of the conditional density is now straightforward. 
Corollary 3.4. If $\left(\mathrm{V}_{1}, \ldots, \mathrm{V}_{\mathrm{n}}\right) \sim \mathrm{KD}_{\mathrm{p}}^{\mathrm{II}}\left(\alpha_{1}, \ldots, \alpha_{\mathrm{n}}, \gamma, \Xi\right)$, then the density of $\mathrm{V}_{i}, i=1, \ldots, \mathrm{n}$ is given by

$$
\begin{aligned}
\Gamma_{p}\left(\sum_{j=1(\neq i)}^{n} \alpha_{j}\right) K_{2}\left(\alpha_{i}, \sum_{j=1(\neq i)}^{n} \alpha_{j}, \gamma, \Xi\right) \operatorname{etr}\left(-\Xi V_{i}\right) \\
\quad \times \operatorname{det}\left(V_{i}\right)^{\alpha_{i}-(p+1) / 2} \operatorname{det}\left(I_{p}+V_{i}\right)^{-\gamma+\sum_{j=1(\neq i)}^{n} \alpha_{j}} \\
\quad \times \Psi\left(\sum_{j=1(\neq i)}^{n} \alpha_{j}, \sum_{j=1(\neq i)}^{n} \alpha_{j}-\gamma+\frac{p+1}{2} ; \Xi\left(I_{p}+V_{i}\right)\right), \quad V_{i}>0
\end{aligned}
$$

Note that the marginal density of $V_{i}$ differs from the Kummer-Gamma density. It is a pdf with an additional factor containing confluent hypergeometric function $\Psi$.

Theorem 3.5. Let $\left(\mathrm{U}_{1}, \ldots, \mathrm{U}_{n}\right) \sim \mathrm{KD}_{p}^{\mathrm{I}}\left(\alpha_{1}, \ldots, \alpha_{n}, \beta, \mathrm{I}_{p}\right)$ and define

$$
W_{i}=\left(I_{p}-\sum_{i=1}^{m} u_{i}\right)^{-1 / 2} u_{i}\left(I_{p}-\sum_{i=1}^{m} u_{i}\right)^{-1 / 2}, \quad i=m+1, \ldots, n
$$

Then the joint density of $\left(W_{m+1}, \ldots, W_{n}\right)$ is given by

$$
\begin{aligned}
& \frac{\Gamma_{p}\left(\sum_{j=m+1}^{n} \alpha_{j}+\beta\right)}{\prod_{i=m+1}^{n} \Gamma_{p}\left(\alpha_{i}\right) \Gamma_{p}(\beta)}\left\{{ }_{1} F_{1}\left(\sum_{i=1}^{n} \alpha_{i} ; \sum_{i=1}^{n} \alpha_{i}+\beta ;-I_{p}\right)\right\}^{-1} \\
& \quad \times \operatorname{etr}\left(-\sum_{i=m+1}^{n} W_{i}\right) \\
& \quad \times \prod_{i=m+1}^{n} \operatorname{det}\left(W_{i}\right)^{\alpha_{i}-(p+1) / 2} \operatorname{det}\left(I_{p}-\sum_{i=m+1}^{n} W_{i}\right)^{\beta-(p+1) / 2} \\
& \quad \times{ }_{1} F_{1}\left(\sum_{i=1}^{m} \alpha_{i} ; \sum_{j=1}^{n} \alpha_{j}+\beta ;-\left(I_{p}-\sum_{i=m+1}^{n} W_{i}\right)\right) \\
& 0<W_{i}<I_{p}, \sum_{i=1}^{m} W_{i}<I_{p} .
\end{aligned}
$$

Proof. Transforming $W_{i}=\left(I_{p}-\sum_{i=1}^{m} u_{i}\right)^{-1 / 2} u_{i}\left(I_{p}-\sum_{i=1}^{m} u_{i}\right)^{-1 / 2}, i=$ $\mathrm{m}+1, \ldots, \mathrm{n}$ with Jacobian $\mathrm{J}\left(\mathrm{U}_{\mathrm{m}+1}, \ldots, \mathrm{U}_{\mathrm{n}} \rightarrow \mathrm{W}_{\mathrm{m}+1}, \ldots, \mathrm{W}_{\mathrm{n}}\right)=\operatorname{det}\left(\mathrm{I}_{\mathrm{p}}-\right.$ 
$\left.\sum_{i=1}^{m} U_{i}\right)^{(n-m)(p+1) / 2}$, in the joint density of $\left(U_{1}, \ldots, U_{n}\right)$, we get

$$
\begin{gathered}
\mathrm{K}_{1}\left(\alpha_{1}, \ldots, \alpha_{n}, \beta, \mathrm{I}_{\mathfrak{p}}\right) \operatorname{etr}\left[-\sum_{i=m+1}^{n} \mathrm{~W}_{i}-\left(\sum_{i=1}^{m} \mathrm{U}_{i}\right)\left(\mathrm{I}_{p}-\sum_{i=m+1}^{n} \mathrm{~W}_{i}\right)\right] \\
\times \prod_{i=1}^{m} \operatorname{det}\left(\mathrm{U}_{i}\right)^{\alpha_{i}-(\mathrm{p}+1) / 2} \operatorname{det}\left(\mathrm{I}_{p}-\sum_{i=1}^{m} \mathrm{U}_{i}\right)^{\sum_{j=m+1}^{n} \alpha_{j}+\beta-(p+1) / 2} \\
\times \prod_{i=m+1}^{n} \operatorname{det}\left(\mathrm{W}_{i}\right)^{\alpha_{i}-(\mathrm{p}+1) / 2} \operatorname{det}\left(\mathrm{I}_{p}-\sum_{i=m+1}^{n} W_{i}\right)^{\beta-(p+1) / 2} \\
0<\mathrm{U}_{i}<\mathrm{I}_{p}, i=m+1, \ldots, n, \sum_{i=m+1}^{n} \mathrm{U}_{i}<\mathrm{I}_{p} \\
0<\mathrm{W}_{i}<\mathrm{I}_{p}, i=1, \ldots, m, \sum_{i=1}^{m} W_{i}<\mathrm{I}_{p} .
\end{gathered}
$$

Now, integrating $\mathrm{U}_{1}, \ldots, \mathrm{U}_{\mathrm{m}}$,

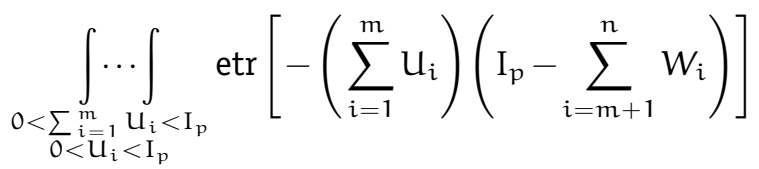

$$
\begin{aligned}
& \times \prod_{i=1}^{m} \operatorname{det}\left(u_{i}\right)^{\alpha_{i}-(p+1) / 2} \\
& \times \operatorname{det}\left(\mathrm{I}_{p}-\sum_{i=1}^{m} \mathrm{U}_{i}\right)^{\sum_{i=m+1}^{n} \alpha_{i}+\beta-(p+1) / 2} \prod_{i=1}^{m} \mathrm{du}_{i} \\
& =\frac{\prod_{i=1}^{m} \Gamma_{p}\left(\alpha_{i}\right)}{\Gamma_{p}\left(\sum_{i=1}^{m} \alpha_{i}\right)} \int_{0<U<I_{p}} \operatorname{etr}\left[-\left(I_{p}-\sum_{i=m+1}^{n} W_{i}\right) \mathrm{U}\right] \\
& \times \operatorname{det}(\mathrm{U})^{\sum_{i=1}^{m} \alpha_{i}-(p+1) / 2} \\
& \times \operatorname{det}\left(\mathrm{I}_{\mathrm{p}}-\mathrm{U}\right)^{\sum_{i=\mathrm{m}+1}^{\mathrm{n}} \alpha_{i}+\beta-(\mathrm{p}+1) / 2} \mathrm{dU} \\
& =\frac{\prod_{i=1}^{m} \Gamma_{p}\left(\alpha_{i}\right) \Gamma_{p}\left(\sum_{i=m+1}^{n} \alpha_{i}+\beta\right)}{\Gamma_{p}\left(\sum_{i=1}^{n} \alpha_{i}+\beta\right)} \\
& \times{ }_{1} F_{1}\left(\sum_{i=1}^{m} \alpha_{i} ; \sum_{i=1}^{n} \alpha_{i}+\beta ;-\left(I_{p}-\sum_{i=m+1}^{n} W_{i}\right)\right) \text {, }
\end{aligned}
$$


and using

$$
\begin{aligned}
& \mathrm{K}_{1}\left(\alpha_{1}, \ldots, \alpha_{n}, \beta, \mathrm{I}_{\mathrm{p}}\right) \frac{\prod_{i=1}^{\mathrm{m}} \Gamma_{\mathrm{p}}\left(\alpha_{i}\right) \Gamma_{\mathrm{p}}\left(\sum_{i=m+1}^{n} \alpha_{i}+\beta\right)}{\Gamma_{p}\left(\sum_{i=1}^{n} \alpha_{i}+\beta\right)} \\
& =\frac{\Gamma_{p}\left(\sum_{i=m+1}^{n} \alpha_{i}+\beta\right)}{\prod_{i=m+1}^{n} \Gamma_{p}\left(\alpha_{i}\right) \Gamma_{p}(\beta)}\left\{{ }_{1} F_{1}\left(\sum_{i=1}^{n} \alpha_{i} ; \sum_{i=1}^{n} \alpha_{i}+\beta ;-I_{p}\right)\right\}^{-1},
\end{aligned}
$$

we get the desired result.

Theorem 3.6. Let $\left(\mathrm{V}_{1}, \ldots, \mathrm{V}_{\mathrm{n}}\right) \sim \mathrm{KD}_{\mathrm{p}}^{\mathrm{II}}\left(\alpha_{1}, \ldots, \alpha_{\mathrm{n}}, \gamma, \mathrm{I}_{\mathrm{p}}\right)$ and define

$$
Z_{i}=\left(I_{p}+\sum_{i=1}^{m} V_{i}\right)^{-1 / 2} V_{i}\left(I_{p}+\sum_{i=1}^{m} V_{i}\right)^{-1 / 2}, \quad i=m+1, \ldots, n
$$

Then the pdf of $\left(Z_{m+1}, \ldots, Z_{n}\right)$ is given by

$$
\begin{aligned}
& \left\{\prod_{i=m+1}^{n} \Gamma_{p}\left(\alpha_{i}\right) \Psi\left(\sum_{i=1}^{n} \alpha_{i}, \sum_{i=1}^{n} \alpha_{i}-\gamma+\frac{p+1}{2} ; I_{p}\right)\right\}^{-1} \\
& \quad \times \operatorname{etr}\left(-\sum_{i=m+1}^{n} Z_{i}\right) \prod_{i=m+1}^{n} \operatorname{det}\left(Z_{i}\right)^{\alpha_{i}-(p+1) / 2} \operatorname{det}\left(I_{p}+\sum_{i=m+1}^{n} Z_{i}\right)^{-\gamma} \\
& \quad \times \Psi\left(\sum_{i=1}^{m} \alpha_{i}, \sum_{i=1}^{n} \alpha_{i}-\gamma+\frac{p+1}{2} ;\left(I_{p}+\sum_{i=m+1}^{n} Z_{i}\right)\right), \quad Z_{i}>0 .
\end{aligned}
$$

Proof. The proof is similar to the proof of Theorem 3.5.

Theorem 3.7. Let $\left(\mathrm{U}_{1}, \ldots, \mathrm{U}_{\mathrm{n}}\right) \sim \mathrm{KD}_{\mathrm{p}}^{\mathrm{I}}\left(\alpha_{1}, \ldots, \alpha_{n}, \beta, \Lambda\right)$ and define $\mathrm{u}=$ $\sum_{i=1}^{n} U_{i}$ and $X_{i}=u^{-1 / 2} u_{i} U^{-1 / 2}, i=1, \ldots, n-1$. Then

(i) $\left(X_{1}, \ldots, X_{n-1}\right)$ and $\mathrm{U}$ are independent,

(ii) $\left(X_{1}, \ldots, X_{n-1}\right) \sim D_{p}^{I}\left(\alpha_{1}, \ldots, \alpha_{n-1} ; \alpha_{n}\right)$, and

(iii) $\mathrm{U} \sim \mathrm{KB}_{\mathrm{p}}\left(\sum_{i=1}^{n} \alpha_{i}, \beta, \Lambda\right)$.

Proof. Substituting $\mathrm{U}_{\mathrm{i}}=\mathrm{U}^{1 / 2} \mathrm{X}_{\mathrm{i}} \mathrm{U}^{1 / 2}, \mathrm{i}=1, \ldots, \mathrm{n}-1$ and $\mathrm{U}_{\mathrm{n}}=\mathrm{U}^{1 / 2}\left(\mathrm{I}_{\mathrm{p}}-\right.$ $\left.\sum_{i=1}^{n-1} X_{i}\right) U^{1 / 2}$ with the Jacobian $J\left(U_{1}, \ldots, U_{n-1}, U_{n} \rightarrow X_{1}, \ldots, X_{n-1}, U\right)=$ 
$\operatorname{det}(U)^{(n-1)(p+1) / 2}$ in the joint density of $\left(U_{1}, \ldots, U_{n}\right)$, we get the desired result.

Theorem 3.8. Let $\left(\mathrm{V}_{1}, \ldots, \mathrm{V}_{\mathrm{n}}\right) \sim \mathrm{KD}_{\mathrm{p}}^{\mathrm{II}}\left(\alpha_{1}, \ldots, \alpha_{n}, \gamma, \Xi\right)$ and define $\mathrm{V}=$ $\sum_{i=1}^{n} V_{i}$ and $Y_{i}=V^{-1 / 2} V_{i} V^{-1 / 2}, i=1, \ldots, n-1$. Then

(i) $\left(Y_{1}, \ldots, Y_{n-1}\right)$ and $V$ are independent,

(ii) $\left(Y_{1}, \ldots, Y_{n-1}\right) \sim D_{p}^{I}\left(\alpha_{1}, \ldots, \alpha_{n-1} ; \alpha_{n}\right)$, and

(iii) $\mathrm{V} \sim \mathrm{KG}_{\mathrm{p}}\left(\sum_{i=1}^{\mathrm{n}} \alpha_{i}, \gamma, \Xi\right)$.

Proof. The proof is similar to the proof of Theorem 3.7.

In Theorems 3.9 and 3.10, we derive the joint pdfs of partial sums of random matrices distributed as matrix variate Kummer-Dirichlet type I or II.

Theorem 3.9. Let $\left(\mathrm{U}_{1}, \ldots, \mathrm{U}_{n}\right) \sim \mathrm{KD}_{\mathrm{p}}^{\mathrm{I}}\left(\alpha_{1}, \ldots, \alpha_{n}, \beta, \Lambda\right)$ and define

$u_{(i)}=\sum_{j=n_{i-1}^{*}+1}^{n_{i}^{*}} u_{j}, \quad \alpha_{(i)}=\sum_{j=n_{i-1}^{*}+1}^{n_{i}^{*}} \alpha_{j}, \quad n_{0}^{*}=0, n_{i}^{*}=\sum_{j=1}^{i} n_{j}, i=1, \ldots, \ell$.

Then $\left(\mathrm{U}_{(1)}, \ldots, \mathrm{U}_{(\ell)}\right) \sim \mathrm{KD}_{\mathrm{p}}^{\mathrm{I}}\left(\alpha_{(1)}, \ldots, \alpha_{(\ell)}, \beta, \Lambda\right)$.

Proof. Make the transformation

$$
\mathrm{u}_{(i)}=\sum_{j=n_{i-1}^{*}+1}^{n_{i}^{*}} \mathrm{u}_{j}, \quad \mathrm{w}_{j}=\mathrm{u}_{(\mathrm{i})}^{-1 / 2} \mathrm{u}_{j} \mathrm{u}_{(\mathrm{i})}^{-1 / 2}
$$

$j=n_{i-1}^{*}+1, \ldots, n_{i}^{*}-1, i=1, \ldots, \ell$. The Jacobian of this transformation is given by

$$
\begin{aligned}
& \mathrm{J}\left(\mathrm{U}_{1}, \ldots, \mathrm{u}_{\mathrm{n}} \longrightarrow \mathrm{W}_{1}, \ldots, \mathrm{W}_{\mathrm{n}_{1}-1}, \mathrm{U}_{(1)}, \ldots, \mathrm{W}_{\mathrm{n}_{\ell-1}^{*}+1}, \ldots, \mathrm{W}_{\mathrm{n}-1}, \mathrm{u}_{(\ell)}\right) \\
& =\prod_{i=1}^{\ell} \mathrm{J}\left(\mathrm{u}_{\mathrm{n}_{i-1}^{*}+1}, \ldots, \mathrm{u}_{\mathrm{n}_{\mathrm{i}}^{*}} \longrightarrow \mathrm{W}_{\mathrm{n}_{\mathrm{i}-1}^{*}+1}, \ldots, \mathrm{W}_{\mathrm{n}_{\mathrm{i}}^{*}-1}, \mathrm{u}_{(\mathrm{i})}\right) \\
& =\prod_{i=1}^{\ell} \operatorname{det}\left(\mathrm{u}_{(i)}\right)^{\left(n_{i}-1\right)(p+1) / 2}
\end{aligned}
$$

Now, substituting from (3.26) and (3.27) in the joint density of $\left(\mathrm{U}_{1}, \ldots, \mathrm{U}_{\mathrm{n}}\right)$ given by $(2.5)$, we get the joint density of $W_{n_{i-1}^{*}+1}, \ldots, W_{n_{i}^{*}-1}, U_{(i)}$, 
where $i=1, \ldots, \ell$, as

$$
\begin{aligned}
\mathrm{K}_{1}\left(\alpha_{1}, \ldots, \alpha_{n}, \beta, \Lambda\right) \operatorname{etr}\left(-\Lambda \sum_{i=1}^{\ell} \mathrm{U}_{(i)}\right) \\
\quad \times \prod_{i=1}^{\ell} \operatorname{det}\left(\mathrm{U}_{(\mathrm{i})}\right)^{\alpha_{(i)}-(p+1) / 2} \operatorname{det}\left(\mathrm{I}_{p}-\sum_{i=1}^{\ell} \mathrm{U}_{(i)}\right)^{\beta-(p+1) / 2} \\
\times \prod_{i=1}^{\ell}\left\{\prod_{j=n_{i-1}^{*}+1}^{n_{i}^{*}-1} \operatorname{det}\left(W_{j}\right)^{\alpha_{j}-(p+1) / 2}\right. \\
\left.\times \operatorname{det}\left(\mathrm{I}_{p}-\sum_{j=n_{i-1}^{*}+1}^{n_{i}^{*}-1} W_{j}\right)^{\alpha_{n_{i}^{*}-(p+1) / 2}}\right\},
\end{aligned}
$$

where $0<\mathrm{U}_{(i)}<\mathrm{I}_{\mathrm{p}}, \sum_{i=1}^{\ell} \mathrm{U}_{(\mathrm{i})}<\mathrm{I}_{\mathrm{p}}, 0<\mathrm{W}_{\mathrm{j}}<\mathrm{I}_{\mathrm{p}}, \mathrm{j}=\mathrm{n}_{\mathrm{i}-1}^{*}+1, \ldots, \mathrm{n}_{\mathrm{i}}^{*}-$ $1, \sum_{j=n_{i-1}^{*}+1}^{n_{i}^{*}-1} W_{j}<I_{p}, i=1, \ldots, \ell$. From (3.28), it is easy to see that $\left(\mathrm{U}_{(1)}, \ldots, \mathrm{U}_{(\ell)}\right)$ and $\left(\mathrm{W}_{\mathrm{n}_{\mathrm{i}-1}^{*}+1}, \ldots, \mathrm{W}_{\mathrm{n}_{\mathrm{i}}^{*}-1}\right), i=1, \ldots, \ell$, are independently distributed. Further, $\left(\mathrm{U}_{(1)}, \ldots, \mathrm{U}_{(\ell)}\right) \sim \mathrm{KD}_{\mathrm{p}}^{\mathrm{I}}\left(\alpha_{(1)}, \ldots, \alpha_{(\ell)}, \beta, \Lambda\right)$ and $\left(\mathrm{W}_{\mathrm{n}_{\mathrm{i}-1}^{*}+1}\right.$, $\left.\ldots, W_{n_{i}^{*}-1}\right) \sim D_{p}^{I}\left(\alpha_{n_{i-1}^{*}+1}, \ldots, \alpha_{n_{i}^{*}-1} ; \alpha_{n_{i}^{*}}\right)$, where $i=1, \ldots, \ell$.

When $\ell=1, \sum_{i=1}^{n} U_{i} \sim K_{p}\left(\sum_{i=1}^{n} \alpha_{i}, \beta, \Lambda\right)$.

Theorem 3.10. Let $\left(\mathrm{V}_{1}, \ldots, \mathrm{V}_{\mathrm{n}}\right) \sim \mathrm{KD}_{\mathrm{p}}^{\mathrm{II}}\left(\alpha_{1}, \ldots, \alpha_{n}, \gamma, \Xi\right)$ and define

$$
V_{(i)}=\sum_{j=n_{i-1}^{*}+1}^{n_{i}^{*}} V_{j}, \quad \alpha_{(i)}=\sum_{j=n_{i-1}^{*}+1}^{n_{i}^{*}} \alpha_{j}, \quad n_{0}^{*}=0, n_{i}^{*}=\sum_{j=1}^{i} n_{j}, i=1, \ldots, \ell .
$$

Then $\left(\mathrm{V}_{(1)}, \ldots, \mathrm{V}_{(\ell)}\right) \sim \mathrm{KD}_{\mathrm{p}}^{\mathrm{II}}\left(\alpha_{(1)}, \ldots, \alpha_{(\ell)}, \gamma, \Xi\right)$.

Proof. Make the transformation

$$
V_{(i)}=\sum_{j=n_{i-1}^{*}+1}^{n_{i}^{*}} V_{j}, \quad Z_{j}=V_{(i)}^{-1 / 2} V_{j} V_{(i)}^{-1 / 2}
$$

where $j=n_{i-1}^{*}+1, \ldots, n_{i}^{*}-1, i=1, \ldots, \ell$. The Jacobian of this transformation 
is given by

$$
\begin{aligned}
& J\left(V_{1}, \ldots, V_{n} \longrightarrow Z_{1}, \ldots, Z_{n_{1}-1}, V_{(1)}, \ldots, Z_{n_{\ell-1}^{*}+1}, \ldots, Z_{n-1}, V_{(\ell)}\right) \\
& =\prod_{i=1}^{\ell} J\left(V_{n_{i-1}^{*}+1}, \ldots, V_{n_{i}^{*}} \longrightarrow Z_{n_{i-1}^{*}+1}, \ldots, Z_{n_{i}^{*}-1}, V_{(i)}\right) \\
& =\prod_{i=1}^{\ell} \operatorname{det}\left(V_{(i)}\right)^{\left(n_{i}-1\right)(p+1) / 2} .
\end{aligned}
$$

Now, substituting from (3.30) and (3.31) in the joint density of $\left(V_{1}, \ldots, V_{n}\right)$ given by (2.6), it can easily be shown that $\left(V_{(1)}, \ldots, V_{(\ell)}\right)$ and $\left(Z_{n_{i-1}^{*}+1}, \ldots\right.$, $\left.Z_{n_{i}^{*}-1}\right), i=1, \ldots, \ell$, are independently distributed. Further, $\left(V_{(1)}, \ldots, V_{(\ell)}\right) \sim$ $\operatorname{KD}_{\mathrm{p}}^{\mathrm{II}}\left(\alpha_{(1)}, \ldots, \alpha_{(\ell)}, \gamma, \Xi\right)$ and $\left(Z_{n_{i-1}^{*}+1}, \ldots, Z_{n_{i}^{*}-1}\right) \sim \mathrm{D}_{\mathrm{p}}^{\mathrm{I}}\left(\alpha_{n_{i-1}^{*}+1}, \ldots\right.$, $\left.\alpha_{n_{i}^{*}-1} ; \alpha_{n_{i}^{*}}\right), i=1, \ldots, \ell$.

When $\ell=1$, the distribution of $\sum_{i=1}^{n} V_{i}$ is Kummer-Gamma with parameters $\sum_{i=1}^{n} \alpha_{i}, \gamma$ and $\Xi$. From the joint density of $\mathrm{U}_{1}, \ldots, \mathrm{U}_{\mathrm{n}}$, we have

$$
\begin{aligned}
& E\left[\prod_{i=1}^{n} \operatorname{det}\left(u_{i}\right)^{r_{i}}\right]
\end{aligned}
$$

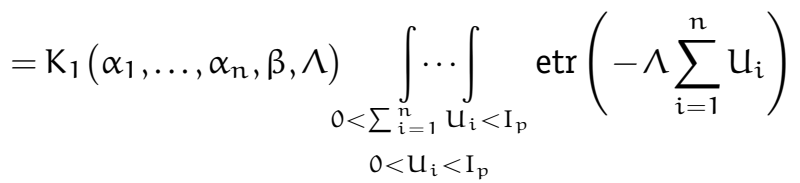

$$
\begin{aligned}
& \times \prod_{i=1}^{n} \operatorname{det}\left(U_{i}\right)^{\alpha_{i}+r_{i}-(p+1) / 2} \operatorname{det}\left(I_{p}-\sum_{i=1}^{n} U_{i}\right)^{\beta-(p+1) / 2} \prod_{i=1}^{n} d u_{i} \\
& =\frac{K_{1}\left(\alpha_{1}, \ldots, \alpha_{n}, \beta, \Lambda\right)}{K_{1}\left(\alpha_{1}+r_{1}, \ldots, \alpha_{n}+r_{n}, \beta, \Lambda\right)}, \quad \operatorname{Re}\left(\alpha_{i}+r_{i}\right)>\frac{p-1}{2} \\
& =\frac{\prod_{i=1}^{n} \Gamma_{p}\left(\alpha_{i}+r_{i}\right) \Gamma_{p}\left(\sum_{i=1}^{n} \alpha_{i}+\beta\right)}{\prod_{i=1}^{n} \Gamma_{p}\left(\alpha_{i}\right) \Gamma_{p}\left[\sum_{i=1}^{n}\left(\alpha_{i}+r_{i}\right)+\beta\right]} \\
& \times \frac{{ }_{1} F_{1}\left(\sum_{i=1}^{n}\left(\alpha_{i}+r_{i}\right) ; \sum_{i=1}^{n}\left(\alpha_{i}+r_{i}\right)+\beta ;-\Lambda\right)}{{ }_{1} F_{1}\left(\sum_{i=1}^{n} \alpha_{i} ; \sum_{i=1}^{n} \alpha_{i}+\beta ;-\Lambda\right)}, \\
& \operatorname{Re}\left(\alpha_{i}+r_{i}\right)>\frac{p-1}{2},
\end{aligned}
$$


where the last step has been obtained using (2.7). Further

$$
\begin{aligned}
& E\left[\operatorname{det}\left(I_{p}-\sum_{i=1}^{n} U_{i}\right)^{h}\right] \\
& =K_{1}\left(\alpha_{1}, \ldots, \alpha_{n}, \beta, \Lambda\right) \int_{\substack{n \\
i=1 \\
u_{i}>0}} \ldots \int_{\substack{\mathrm{U}_{i}<\mathrm{I}_{p} \\
\mathrm{U}_{i=1}}} \operatorname{etr}\left(-\Lambda \sum_{i=1}^{n} \mathrm{u}_{i}\right) \\
& \times \prod_{i=1}^{n} \operatorname{det}\left(U_{i}\right)^{\alpha_{i}-(p+1) / 2} \operatorname{det}\left(I_{p}-\sum_{i=1}^{n} U_{i}\right)^{\beta+h-(p+1) / 2} \prod_{i=1}^{n} d U_{i} \\
& =\frac{K_{1}\left(\alpha_{1}, \ldots, \alpha_{n}, \beta, \Lambda\right)}{K_{1}\left(\alpha_{1}, \ldots, \alpha_{n}, \beta+h, \Lambda\right)}, \quad \operatorname{Re}(h)>-\beta+\frac{p-1}{2} \\
& =\frac{\Gamma_{p}(\beta+h) \Gamma_{p}\left(\sum_{i=1}^{n} \alpha_{i}+\beta\right)}{\Gamma_{p}(\beta) \Gamma_{p}\left(\sum_{i=1}^{n} \alpha_{i}+\beta+h\right)} \frac{F_{1}\left(\sum_{i=1}^{n} \alpha_{i} ; \sum_{i=1}^{n} \alpha_{i}+\beta+h ;-\Lambda\right)}{{ }_{1} F_{1}\left(\sum_{i=1}^{n} \alpha_{i} ; \sum_{i=1}^{n} \alpha_{i}+\beta ;-\Lambda\right)}, \\
& \operatorname{Re}(h)>-\beta+\frac{p-1}{2} \text {. }
\end{aligned}
$$

Alternately, the above moment expression can be obtained by noting that $\sum_{i=1}^{n} U_{i}$ has Kummer-Beta distribution. Similarly, for Kummer-Dirichlet type II matrices

$$
\begin{aligned}
& E\left[\prod_{i=1}^{n} \operatorname{det}\left(V_{i}\right)^{r_{i}}\right] \\
& =\prod_{i=1}^{n} \frac{\Gamma_{p}\left(\alpha_{i}+r_{i}\right)}{\Gamma_{p}\left(\alpha_{i}\right)} \frac{\Psi\left(\sum_{i=1}^{n}\left(\alpha_{i}+r_{i}\right), \sum_{i=1}^{n}\left(\alpha_{i}+r_{i}\right)-\gamma+(p+1) / 2 ; \Xi\right)}{\Psi\left(\sum_{i=1}^{n} \alpha_{i}, \sum_{i=1}^{n} \alpha_{i}-\gamma+(p+1) / 2 ; \Xi\right)}, \\
& \operatorname{Re}\left(\alpha_{i}+r_{i}\right)>\frac{p-1}{2} \\
& E\left[\operatorname{det}\left(I_{p}+\sum_{i=1}^{n} V_{i}\right)^{-h}\right]=\frac{\Psi\left(\sum_{i=1}^{n} \alpha_{i}, \sum_{i=1}^{n} \alpha_{i}-\gamma-h+(p+1) / 2 ; \Xi\right)}{\Psi\left(\sum_{i=1}^{n} \alpha_{i}, \sum_{i=1}^{n} \alpha_{i}-\gamma+(p+1) / 2 ; \Xi\right)} .
\end{aligned}
$$

Next two results give certain asymptotic distributions for the KummerDirichlet type I and type II distributions (see Javier and Gupta [4]).

Theorem 3.11. Let $\left(\mathrm{U}_{1}, \ldots, \mathrm{U}_{\mathrm{n}}\right) \sim \mathrm{KD}_{\mathrm{p}}^{\mathrm{I}}\left(\alpha_{1}, \ldots, \alpha_{n}, \beta, \beta \Lambda\right)$ and $\mathrm{W}=\left(\mathrm{W}_{1}, \ldots\right.$, $\left.\mathrm{W}_{\mathrm{n}}\right)$ be defined by $\mathrm{W}_{\mathrm{i}}=\beta \mathrm{U}_{i}, i=1, \ldots, \mathrm{n}$. Then $\mathrm{W}$ is asymptotically distributed as a product of independent matrix variate gamma densities; 
more specifically

$$
\lim _{\beta \rightarrow \infty} f(W)=\prod_{i=1}^{n} \frac{\operatorname{det}\left(W_{i}\right)^{\alpha_{i}-(p+1) / 2} \operatorname{etr}\left[-\left(I_{p}+\Lambda\right) W_{i}\right]}{\operatorname{det}\left(I_{p}+\Lambda\right)^{\alpha_{i}} \Gamma_{p}\left(\alpha_{i}\right)},
$$

where $\mathrm{f}(\mathrm{W})$ denotes the density of the matrix $\mathrm{W}$.

Proof. In the joint density of $\left(\mathrm{U}_{1}, \ldots, \mathrm{U}_{\mathrm{n}}\right)$ given by (2.5) transform $W_{i}=$ $\beta \mathrm{U}_{i}, \quad i=1, \ldots, \mathrm{n}$ with the Jacobian $\mathrm{J}\left(\mathrm{U}_{1}, \ldots, \mathrm{U}_{\mathrm{n}} \rightarrow \mathrm{W}_{1}, \ldots, \mathrm{W}_{\mathrm{n}}\right)=$ $\beta^{-n p(p+1) / 2}$. The density of $W=\left(W_{1}, \ldots, W_{n}\right)$ is given by

$$
\begin{aligned}
f(W)= & \frac{\Gamma_{p}\left(\sum_{i=1}^{n} \alpha_{i}+\beta\right)}{\Gamma_{p}(\beta)} \beta^{-p \sum_{i=1}^{n} \alpha_{i}}\left\{{ }_{1} F_{1}\left(\sum_{i=1}^{n} \alpha_{i} ; \sum_{i=1}^{n} \alpha_{i}+\beta ;-\beta \Lambda\right)\right\}^{-1} \\
& \times \operatorname{etr}\left(-\Lambda \sum_{i=1}^{n} W_{i}\right)\left\{\prod_{i=1}^{n} \frac{\operatorname{det}\left(W_{i}\right)^{\alpha_{i}-(p+1) / 2}}{\Gamma_{p}\left(\alpha_{i}\right)}\right\} \\
& \times \operatorname{det}\left(I_{p}-\frac{1}{\beta} \sum_{i=1}^{n} W_{i}\right)^{\beta-(p+1) / 2} .
\end{aligned}
$$

The result follows, since

$$
\begin{gathered}
\lim _{\beta \rightarrow \infty} \frac{\Gamma_{p}\left(\sum_{i=1}^{n} \alpha_{i}+\beta\right)}{\Gamma_{p}(\beta)} \beta^{-p} \sum_{i=1}^{n} \alpha_{i}=1 \\
\lim _{\beta \rightarrow \infty}{ }_{1} F_{1}\left(\sum_{i=1}^{n} \alpha_{i} ; \sum_{i=1}^{n} \alpha_{i}+\beta ;-\beta \Lambda\right) \\
={ }_{1} F_{0}\left(\sum_{i=1}^{n} \alpha_{i} ;-\Lambda\right)=\operatorname{det}\left(I_{p}+\Lambda\right)^{-\sum_{i=1}^{n} \alpha_{i}}, \\
\lim _{\beta \rightarrow \infty} \operatorname{det}\left(I_{p}-\frac{1}{\beta} \sum_{i=1}^{n} W_{i}\right)^{\beta-(p+1) / 2}=\operatorname{etr}\left(-\sum_{i=1}^{n} W_{i}\right) .
\end{gathered}
$$

An analogous result for Kummer-Dirichlet type II distribution is shown to be the following.

Theorem 3.12. Let $\left(\mathrm{V}_{1}, \ldots, \mathrm{V}_{\mathrm{n}}\right) \sim \mathrm{KD}_{\mathrm{p}}^{\mathrm{II}}\left(\alpha_{1}, \ldots, \alpha_{\mathrm{n}}, \gamma,|\gamma| \Xi\right), \gamma \neq 0$, and $\mathrm{W}=$ $\left(\mathrm{W}_{1}, \ldots, \mathrm{W}_{\mathrm{n}}\right)$ be defined by $\mathrm{W}_{\mathrm{i}}=|\gamma| \mathrm{V}_{\mathrm{i}}, \mathrm{i}=1, \ldots, \mathrm{n}$. Then, $\mathrm{W}$ is asymptotically distributed as a product of independent matrix variate gamma 
densities; more specifically

$$
\lim _{|\gamma| \rightarrow \infty} g(W)=\prod_{i=1}^{n} \frac{\operatorname{det}\left(W_{i}\right)^{\alpha_{i}-(p+1) / 2} \operatorname{etr}\left[-\left(I_{p}+\Xi\right) W_{i}\right]}{\operatorname{det}\left(I_{p}+\Xi\right)^{\alpha_{i}} \Gamma_{p}\left(\alpha_{i}\right)}
$$

where $\mathrm{g}(\mathrm{W})$ denotes the density of the matrix $\mathrm{W}$.

Proof. We prove the result for $\gamma>0$. The proof for $\gamma<0$ follows similar steps. The density of $W=\left(W_{1}, \ldots, W_{n}\right)$ is given by

$$
\begin{aligned}
g(W)= & \gamma^{-p \sum_{i=1}^{n} \alpha_{i}}\left\{\Psi\left(\sum_{i=1}^{n} \alpha_{i}, \sum_{i=1}^{n} \alpha_{i}-\gamma+\frac{p+1}{2} ; \gamma \Xi\right)\right\}^{-1} \\
& \times \operatorname{etr}\left(-\Xi \sum_{i=1}^{n} W_{i}\right)\left\{\prod_{i=1}^{n} \frac{\operatorname{det}\left(W_{i}\right)^{\alpha_{i}-(p+1) / 2}}{\Gamma_{p}\left(\alpha_{i}\right)}\right\} \\
& \times \operatorname{det}\left(I_{p}+\frac{1}{\gamma} \sum_{i=1}^{n} W_{i}\right)^{-\gamma} .
\end{aligned}
$$

The result follows, since

$$
\begin{gathered}
\lim _{\gamma \rightarrow \infty} \gamma^{p \sum_{i=1}^{n} \alpha_{i}} \Psi\left(\sum_{i=1}^{n} \alpha_{i}, \sum_{i=1}^{n} \alpha_{i}-\gamma+\frac{p+1}{2} ; \gamma \Xi\right)=\operatorname{det}\left(I_{p}+\Xi\right)^{-\sum_{i=1}^{n} \alpha_{i}}, \\
\lim _{\gamma \rightarrow \infty} \operatorname{det}\left(I_{p}+\frac{1}{\gamma} \sum_{i=1}^{n} W_{i}\right)^{-\gamma}=\operatorname{etr}\left(-\sum_{i=1}^{n} W_{i}\right) .
\end{gathered}
$$

\section{Acknowledgement}

The research work of Liliam Cardeño and Daya K. Nagar was supported by the Comité para el Desarrollo de la Investigación, Universidad de Antioquia, research grant number IN91CE.

\section{References}

[1] C. Armero and M. J. Bayarri, A Bayesian analysis of a queueing system with unlimited service, J. Statist. Plann. Inference 58 (1997), no. 2, 241-261. CMP 1450 015. Zbl 880.62025.

[2] M. B. Gordy, Computationally convenient distributional assumptions for common-value auctions, Comput. Econom. 12 (1998), 61-78. Zbl 912.90093.

[3] A. K. Gupta and D. K. Nagar, Matrix Variate Distributions, Chapman \& Hall/CRC Monographs and Surveys in Pure and Applied Mathematics, vol. 104, Chapman \& Hall/CRC, Florida, 2000. MR 2001d:62055. Zbl 935.62064. 
[4] W. R. Javier and A. K. Gupta, On generalized matric variate beta distributions, Statistics 16 (1985), no. 4, 549-557. MR 86m:62023. Zbl 602.62039.

[5] D. K. Nagar and L. Cardeño, Matrix variate Kummer-Gamma distribution, Random Oper. Stochastic Equations 9 (2001), no. 3, 207-218.

[6] D. K. Nagar and A. K. Gupta, Matrix variate Kummer-Beta distribution, to appear in J. Austral. Math. Soc.

[7] K. W. Ng and S. Kotz, Kummer-Gamma and Kummer-Beta univariate and multivariate distributions, Research report, Department of Statistics, The University of Hong Kong, Hong Kong, 1995.

Arjun K. Gupta: Department of Mathematics and Statistics, Bowling Green State University, Bowling Green, OH 43403-0221, USA

Liliam Cardeño and Daya K. Nagar: Departamento de Matemáticas, Universidad de Antioquia, Medellín, A. A. 1226, Colombia 


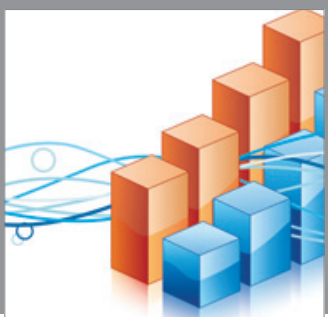

Advances in

Operations Research

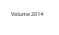

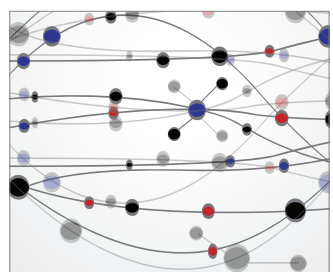

\section{The Scientific} World Journal
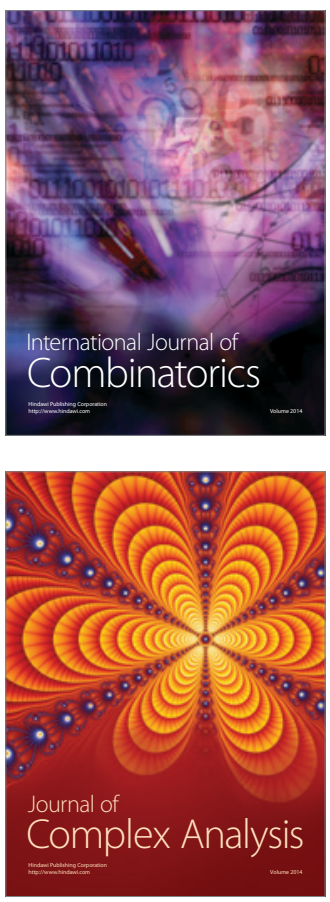

International Journal of

Mathematics and

Mathematical

Sciences
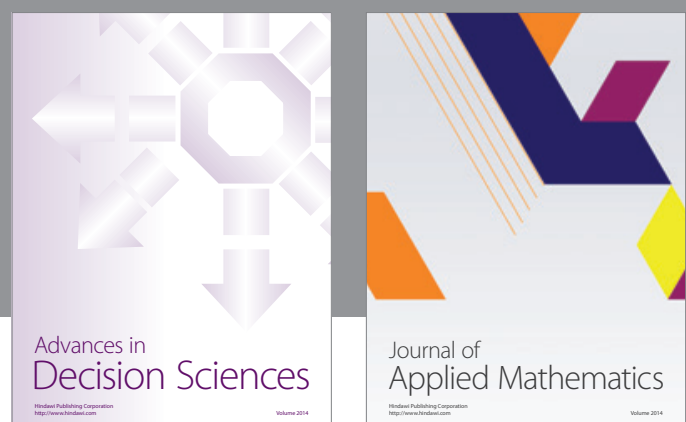

Journal of

Applied Mathematics
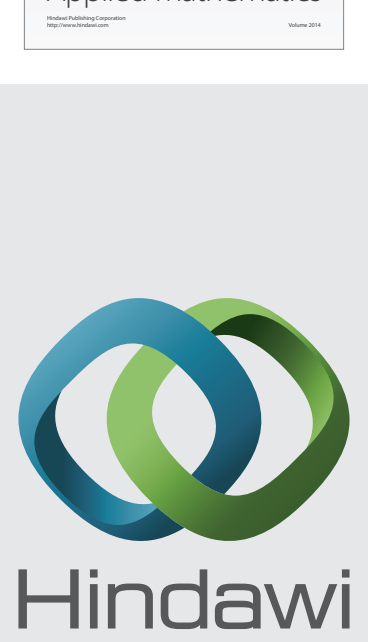

Submit your manuscripts at http://www.hindawi.com
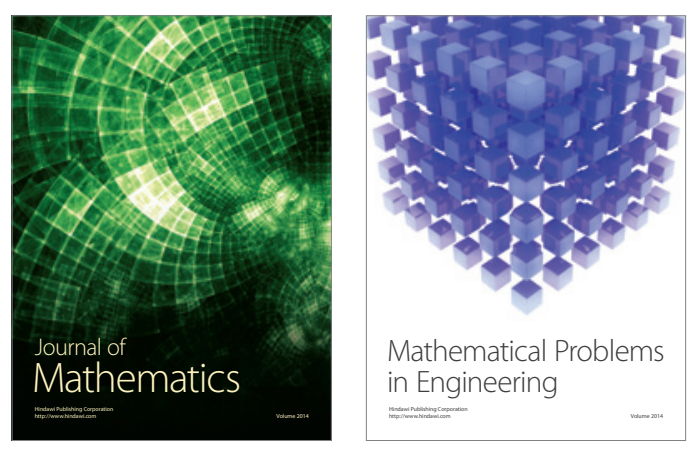

Mathematical Problems in Engineering
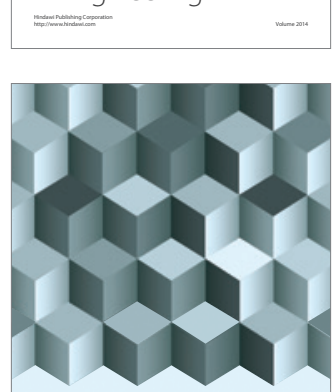

Journal of

Function Spaces
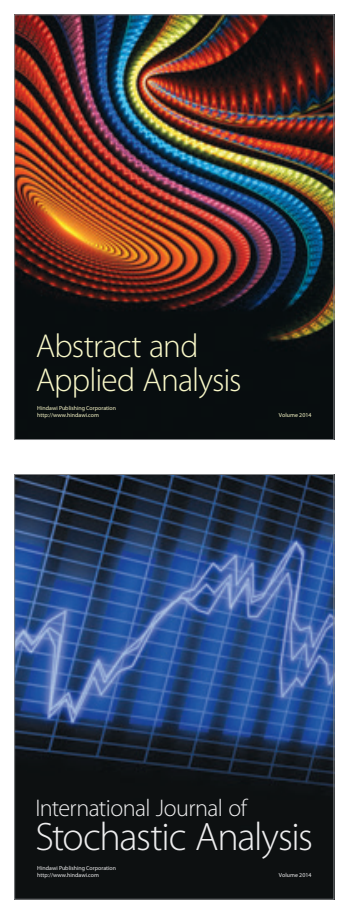

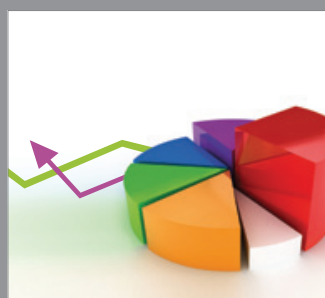

ournal of

Probability and Statistics

Promensencen
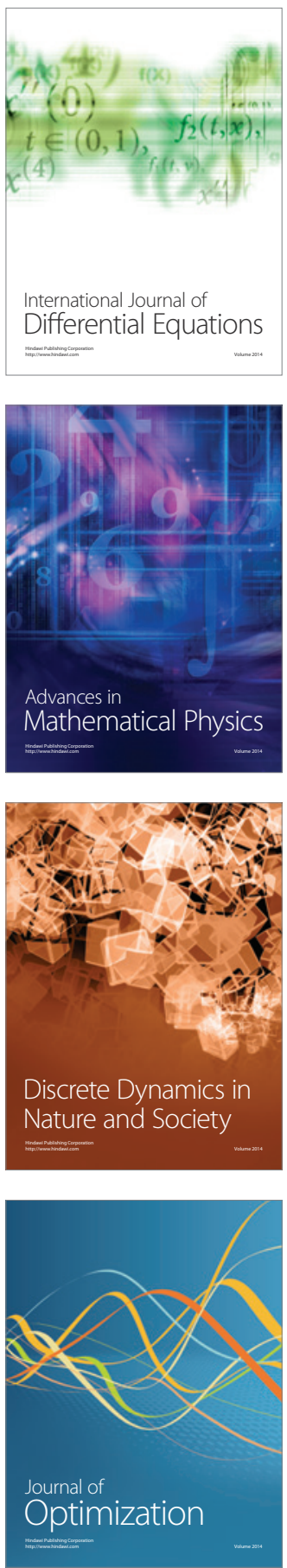